\title{
Research on insulation joint damage of the station track circuit in high speed and heavy load condition
}

\author{
Guanggang $\mathrm{Ji}^{1,2}$, Jianlei Liu ${ }^{1,2}$, a , Zheng $\mathrm{Li}^{1}$ and Guangqian Chu ${ }^{1}$ \\ 1.College of Rail Transportation, Shandong Jiaotong University, Jinan 250000, China \\ 2. Rail Transit Safety Technology and Equipment Laboratory, Key Laboratory of transportation industry of Shandong Province, Jinan 250000 , \\ China
}

\begin{abstract}
In high-speed and heavy-load operation environment, the insulation joints of the station track circuit are damaged in many stations, which caused the carrier information of the adjacent sections to interfere with each other, endangered the safety of the train and reduced the reliability of the track circuit. In order to solve the incorrect code problem caused by the insulation joint breakage, we analysed the insulation damage situation for the station track circuit and the structural principle of BES choke transformer. Then the different structures of the insulation damage protection circuit are given and the relevant parameters are obtained, and verifying the anti-interference ability of the new BES choke transformer. Finally, a complete four-terminal network model of the new BES choke transformer is established. And its four-terminal network parameters are calculated by matlab simulation, which provides the theoretical basis for establishing the track circuit complete system.
\end{abstract}

\section{Introduction}

The ZPW-2000 track circuit is an important component of the train control system, which has the ability to transmit train information, train positioning and other main functions ${ }^{[1]}$. The section line adopts ZPW-2000 series non-insulated track circuits, due to the railways continuous development towards high speed and heavy load, in order to ensure the continuity of train control information, the section and station are adopt the same standard track circuit in high speed and heavy load situation, that is, the ZPW-2000 integrated track circuit ${ }^{[2]}$. When the ZPW-2000 track circuit is applied to the station, the non-insulated track circuit is not suitable. The insulated track circuit must be adopted, using the mechanical insulation joint to divide the adjacent track circuit section. The choke transformer is used to conduct the traction current and ensure the reliable transmission of frequency-shift signal in the mechanical insulation joint. The insulated track circuit in station is shown in Figure 1.

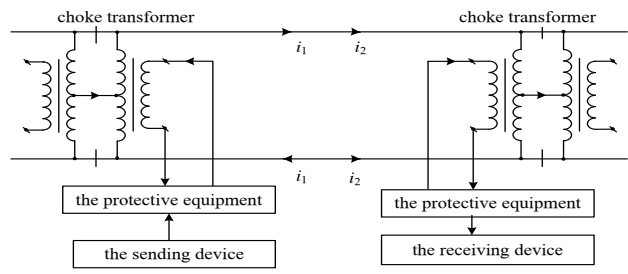

Figure 1. Structure of the insulated track circuit in station

As one of the key equipment of the station track a Corresponding author: 1425252655@qq.com circuit, the insulation joint plays an important role in the auxiliary positioning of the train and the isolation of the adjacent section frequency shift signals ${ }^{[3]}$. It is easy to produce arcing phenomenon when the wheel set of high-speed train passes through ${ }^{[4]}$, which leads to serious burning loss of insulating joint and rail.

In China high-speed operation lines, such as Beijing-Shanghai high-speed railway and so on, have suffered burnout of mechanical insulation joints and occurred more than 300 times, which seriously affecting the normal operation of high-speed railway ${ }^{[5]}$. In view of the burning damage of mechanical insulation joint in high-speed railway passenger stations, the domestic scholars mainly focuses on that how to alleviate the burning damage of mechanical insulation joint ${ }^{[6]}$, and did not give specific solutions to the problem of the carrier frequency mutual interference of the adjacent track circuit section in station. Based on the improvement of the traditional choke transformer, this paper proposes a kind of rail-side protection scheme that can effectively prevent the carrier frequency mutual interference of the adjacent track circuit section caused by the mechanical insulation damage in station.

\section{Analysis of insulation joint damage}

When the station track circuit use the insulation section to divide the adjacent track section, if the insulation section has unilateral or bilateral insulation damage and iron filings, the signal of the adjacent 
section will be transmitted across the zone, causing the vehicle equipment receive the mistake code, thus endangering the train safety ${ }^{[7]}$. The flow direction of the adjacent section signal current in current track circuit is shown in Figure 2.

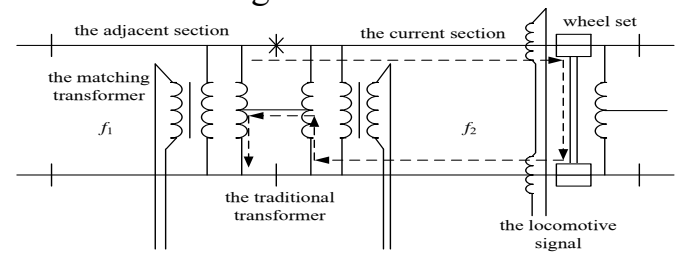

Figure 2. Signal current cross-region transmission diagram

We take a third-line station as an example. The two sides of the station are the main lines, and the middle is the third line with running in two directions. According to the carrier frequency configuration principle, we can know that no matter how it is arranged, the carrier frequency of the third line will be the same as one of the main lines. According to one of the situations, the station carrier frequency configuration is shown in Figure 3.

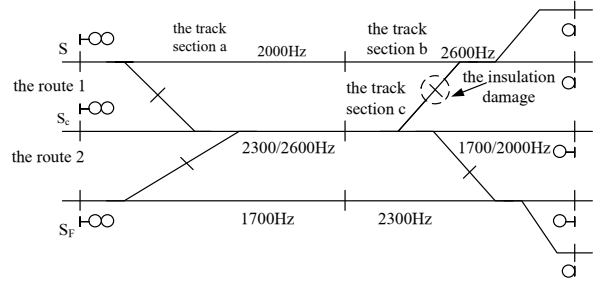

Figure 3. The third-line station structure

Now the two trains are running in the uplink direction of the route 1 and 2 respectively. When the train runs in the track section a, the insulation joint between the section $\mathrm{b}$ and $\mathrm{c}$ is damaged for some reason, so the carrier frequency information of section $c$ will be transmitted to section $b$. When the train runs to section b, the on-board equipment will use $2000 \mathrm{~Hz}$ carrier frequency information of section c as effective information and ignore $2600 \mathrm{~Hz}$ carrier frequency information of section $b$, which threatens the train safety. In the long-term application of the signal system, we must find reasonable solution to ensure the railway operation is an inevitable requirement.

\section{Structure of BES choke transformer}

The track circuit of the high-speed and heavy-load stations in China adopts the insulated track circuit system, and a choke transformer is used at the mechanical insulation joint to conduct the traction current of the adjacent track section. In order to adapt to the new transportation requirements and meet the new anti-interference requirements of the signal system, at present, The BES choke transformer is generally used in high-speed and heavy-duty railway stations in China. The structure of BES choke transformer is shown in Figure 4.

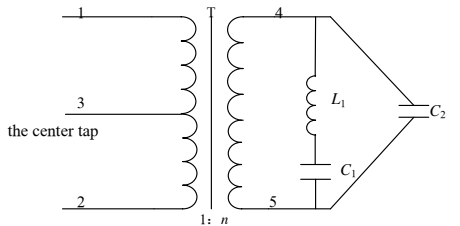

Figure 4. Structure of BES choke transformer

The $50 \mathrm{~Hz}$ series resonant circuit composed of $\mathrm{L}_{1} \mathrm{C}_{1}$ in the adapter part is used to reduce interference energy. The lifting capacitor $\mathrm{C}_{2}$, the $50 \mathrm{~Hz}$ series resonant circuit and the equivalent inductance of the choke transformer secondary side coil together form a parallel resonance of the frequency-shifted signal, showing high impedance and the frequency-shifted signal impedance value converted to the primary side of the choke transformer is more than $17 \Omega$, which can meet the anti-interference requirements and ensure the reliable transmission of frequency-shifted signal. Where $\mathrm{n}=30, \mathrm{C}_{1}=30 \mu \mathrm{F}, \mathrm{L}_{1}=0.338 \mathrm{H}^{[8]}$, the value of $\mathrm{C}_{2}$ is related to the track section carrier frequency, the $\mathrm{C}_{2}$ values corresponding to $1700 \mathrm{~Hz}, 2000 \mathrm{~Hz}, 2300 \mathrm{~Hz}$, and $2600 \mathrm{~Hz}$ are $31.10 \mathrm{nF}, 22.47 \mathrm{nF}, 16.98 \mathrm{nF}, 13.29 \mathrm{nF}$.

\section{Structure of insulation damage protection circuit}

The choke transformer is one of the important equipment of the station track circuit, we proposed to parallel the insulation damage protection circuit on the BES choke transformer secondary side to solve the adjacent section carrier frequency mutual interference.

When the track circuit insulation joint of the high-speed and heavy-load stations is damaged, the series resonance circuit of the insulation damage protection circuit can be used to eliminate the adjacent section carrier frequency interference information. Under normal circumstances, the parallel resonance circuit is used to ensure the signal reliable transmission of the current section. And for on-board equipment, the track circuit always has high reliability and high safety, ensuring the reliable transmission of frequency-shifted signal. Due to the different configuration of the up and down track circuit carrier frequency, the insulation damage protection circuit has different structures for different lines.

\subsection{Insulation damage protection circuit with carrier frequency $1700 / 2000 \mathrm{~Hz}$}

When the insulation joint is damaged for some reason, the $2300 / 2600 \mathrm{~Hz}$ carrier frequency information of the adjacent section will be transmitted to the current section, which can cause interference to $1700 / 2000 \mathrm{~Hz}$ carrier frequency information of the current section. According to the principle of series-parallel resonant circuit, the insulation damage protection circuit structure for $1700 / 2000 \mathrm{~Hz}$ is shown in figure 5 . 


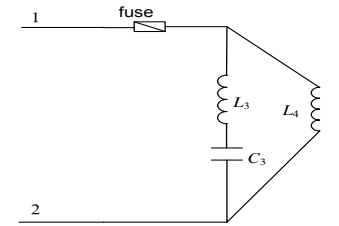

Figure 5. The protection circuit of $1700 / 2000 \mathrm{~Hz}$

When the current section frequency is $1700 \mathrm{~Hz}$, $\mathrm{L}_{3} \mathrm{C}_{3}$ forms series resonance of $2300 \mathrm{~Hz}$, which is used to eliminate $2300 \mathrm{~Hz}$ interference frequency. Since $\mathrm{L}_{3} \mathrm{C}_{3}$ constitutes series resonance of $2300 \mathrm{~Hz}$, it is capacitive to the $1700 \mathrm{~Hz}$ frequency, so through the parallel $\mathrm{L}_{4}$, it is combined with $\mathrm{L}_{3} \mathrm{C}_{3}$ series resonance circuit to form parallel resonance of $1700 \mathrm{~Hz}$, which can ensure that the normal transmission of the current section signal. When the current section frequency is $2000 \mathrm{~Hz}$, the circuit principle is the same as $1700 \mathrm{~Hz}$.

According to the above analysis, the following formula can be obtained:

$$
\begin{aligned}
& \omega_{1}^{2} L_{3} C_{3}=1 \\
& \frac{1}{\omega_{2} C_{3}}-\omega_{2} L_{3}=\omega_{2} L_{4} \\
& L_{3}=\frac{L_{4}}{\left(\frac{f_{1}}{f_{2}}\right)^{2}-1}
\end{aligned}
$$

Take $\mathrm{C}_{3}=85 \mathrm{nF}$, when the current section frequency is $1700 \mathrm{~Hz}$, according to formula (1) and (3), $\mathrm{L}_{3}=$ $56.39 \mathrm{mH}, \mathrm{L}_{4}=45.11 \mathrm{mH}$. When the current section frequency is $2000 \mathrm{~Hz}$, according to the formula, $\mathrm{L}_{3}=$ $44.13 \mathrm{mH}, \mathrm{L}_{4}=44.30 \mathrm{mH}$.

\subsection{Insulation damage protection circuit with carrier frequency $2300 / 2600 \mathrm{~Hz}$}

When the insulation joint is damaged for some reason, the $1700 / 2000 \mathrm{~Hz}$ frequency information will be transmitted to the current section, which can cause interference to $2300 / 2600 \mathrm{~Hz}$ frequency information. According to the principle of series-parallel resonant circuit, the insulation damage protection circuit structure $2300 / 2600 \mathrm{~Hz}$ is shown in figure 6 .

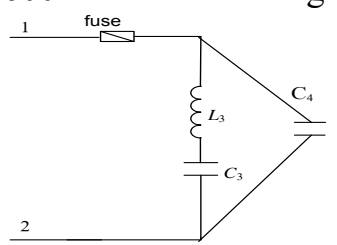

Figure 6. The protection circuit of $2300 / 2600 \mathrm{~Hz}$

When the current section frequency is $2300 \mathrm{~Hz}$, $\mathrm{L}_{3} \mathrm{C}_{3}$ forms series resonance of $1700 \mathrm{~Hz}$, which is used to eliminate the $1700 \mathrm{~Hz}$ interference frequency. Since $\mathrm{L}_{3} \mathrm{C}_{3}$ constitutes series resonance of $1700 \mathrm{~Hz}$, it is capacitive to the $2300 \mathrm{~Hz}$ frequency, so through the parallel capacitance $\mathrm{C}_{4}$, it is combined with $\mathrm{L}_{3} \mathrm{C}_{3}$ series resonance circuit to form parallel resonance of $2300 \mathrm{~Hz}$, which can ensure the current section signal normal transmission. When the current section carrier frequency is $2600 \mathrm{~Hz}$, the circuit principle is the same as $2300 \mathrm{~Hz}$. And the following formula can be obtained:

$$
\begin{gathered}
\omega_{2} L_{3}-\frac{1}{\omega_{2} C_{3}}=\frac{1}{\omega_{2} C_{4}} \\
C_{4}=\frac{C_{3}}{\left(\frac{f_{2}}{f_{1}}\right)^{2}-1}
\end{gathered}
$$

when the current section frequency is $2000 \mathrm{~Hz}$, according to formula (1) and (5), $\mathrm{L}_{3}=96.30 \mathrm{mH}$, $\mathrm{C}_{4}=106.25 \mathrm{nF}$. When the current section carrier frequency is $2600 \mathrm{~Hz}, \mathrm{~L}_{3}=74.60 \mathrm{mH}, \mathrm{C}_{4}=123.20 \mathrm{nF}$.

\subsection{Anti-interference ability analysis of new BES choke transformer}

According to the relevant standards of choke transformer equipment, the input impedance of new BES choke transformer to the frequency-shifted signal is more than $17 \Omega$.

According to the parallel resonant principle:

$$
X_{\mathrm{s} 2}=\frac{Q}{\omega C}=Q \omega L
$$

According to the BES type choke transformer structure, we can obtain equation (7), (8) and (9).

$$
\begin{gathered}
X_{\mathrm{s} 21}=\frac{Q_{1} Q_{2} L_{4} \omega}{Q_{1}+Q_{2} \omega^{2} L_{4} C_{2}} \\
X_{\mathrm{s} 22}=\frac{Q_{1} Q_{2}}{Q_{1} \omega C_{4}+Q_{2} \omega C_{2}} \\
X_{\mathrm{s}}=\frac{X_{\mathrm{s} 2}}{(n / 1)^{2}}
\end{gathered}
$$

According to the simulation study, $Q_{1}=25, Q_{2}=50$, $\omega=2 \pi f$. According to the above formula, the input impedance of new BES type choke transformer for different frequency shift signals are shown in Table 1.

Table 1. The impedance value of different carry frequency

\begin{tabular}{ccccc}
\hline $\begin{array}{c}\text { the carry } \\
\text { frequency }(\mathrm{Hz})\end{array}$ & 1700 & 2000 & 2300 & 2600 \\
$\begin{array}{c}\text { the signal impedance } \\
\text { value }(\Omega)\end{array}$ & 20.27 & 19.67 & 27.47 & 22.73 \\
\hline
\end{tabular}

And the input impedance of new BES choke transformer are greater than $17 \Omega$, which can meet the requirements of anti-interference.

\section{Establishment of four-terminal network model}

According to rail characteristics, the track circuit is equivalent to the transmission line with uniformly distributed parameters. The signal transmission characteristics can be described by four-terminal network ${ }^{[9]}$. Through comparative analysis, the BES choke transformers complete model mainly consists of ideal autotransformer, equivalent excitation impedance, ideal transformer, adapter part, etc ${ }^{[10]}$. And each part is equivalent to the corresponding four-terminal network, as shown in Figure 7, the transmission equation can be expressed as: 


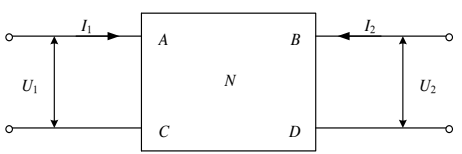

Figure 7. The four-terminal network model

$$
\left[\begin{array}{c}
U_{1} \\
I_{1}
\end{array}\right]=\left[\begin{array}{ll}
A & B \\
C & D
\end{array}\right]\left[\begin{array}{c}
U_{2} \\
I_{2}
\end{array}\right]=N \cdot\left[\begin{array}{c}
U_{2} \\
I_{2}
\end{array}\right]
$$

According to the above analysis, the four-terminal network model of new BES choke transformer with $1700 / 2000 \mathrm{~Hz}$ and $2300 / 2600 \mathrm{~Hz}$ are $\mathrm{N}_{\text {ZPW-X }}$ and $\mathrm{N}_{\text {ZPW-S }}$ respectively.

$$
\begin{aligned}
& N_{\mathrm{ZPW}-\mathrm{X}}=\left(\begin{array}{cc}
1 & 0 \\
\frac{1}{R_{\mathrm{M}}}+\frac{1}{j \omega L_{\mathrm{M}}} & 1
\end{array}\right)\left(\begin{array}{cc}
\frac{1}{n} & 0 \\
0 & n
\end{array}\right)\left(\begin{array}{cc}
1 & 0 \\
\frac{1}{j \omega L_{2}} & 1
\end{array}\right) \\
& \left(\begin{array}{cc}
j \omega C_{2}+\frac{1}{r+j \omega L_{1}+\frac{1}{j \omega C_{1}}} & 1
\end{array}\right)\left(\begin{array}{cc}
\frac{1}{j \omega L_{4}}+\frac{1}{j \omega L_{3}+\frac{1}{j \omega C_{3}}} & 1
\end{array}\right)
\end{aligned}
$$

$$
\begin{aligned}
& N_{\mathrm{ZPW}-\mathrm{S}}=\left(\begin{array}{cc}
1 & 0 \\
\frac{1}{R_{\mathrm{M}}}+\frac{1}{j \omega L_{\mathrm{M}}} & 1
\end{array}\right)\left(\begin{array}{cc}
\frac{1}{n} & 0 \\
0 & n
\end{array}\right) \\
& \left(\begin{array}{cc}
1 & 0 \\
\frac{1}{j \omega L_{2}} & 1
\end{array}\right)\left(\begin{array}{cc}
j \omega C_{2}+\frac{1}{r+j \omega L_{1}+\frac{1}{j \omega C_{1}}} & 1
\end{array}\right) \\
& \left(\begin{array}{cc}
j \omega C_{4}+\frac{1}{j \omega L_{3}+\frac{1}{j \omega C_{3}}} & 1
\end{array}\right)
\end{aligned}
$$

According to the four-terminal network model analysis of the new BES choke transformer, the matlab is used to simulate calculation $\mathrm{N}_{Z P W}$. The four-terminal network parameters of new BES choke transformer are shown in Table 2.

Table 2. The four-terminal network parameters of choke transformer with insulation damage protection circuit

\begin{tabular}{cccccccc}
\hline$C_{2}(\mathrm{nF})$ & $L_{4}(\mathrm{mH})$ & $C_{4}(\mathrm{nF})$ & $\begin{array}{c}\text { carry } \\
\text { frequency } \\
(\mathrm{Hz})\end{array}$ & $A$ & $B$ & $C$ & $D$ \\
\hline 31.10 & 45.11 & $/$ & 1700 & $1 / 30$ & 0 & $0.00070-0.0038 i$ & 30 \\
22.47 & 35.30 & $/$ & 2000 & $1 / 30$ & 0 & $0.00060-0.0142 i$ & 30 \\
16.98 & $/$ & 106.25 & 2300 & $1 / 30$ & 0 & $0.00060-0.0071 i$ & 30 \\
13.29 & $/$ & 123.20 & 2600 & $1 / 30$ & 0 & $0.00060-0.0009 i$ & 30 \\
\hline
\end{tabular}

\section{Conclusion}

Aiming at the problems of the adjacent section track circuit carrier frequency mutual interference caused by the insulation joint damage in station track circuit, this paper put forward the relative rail-side protection scheme. By using the relevant anti-interference indexes, it is verified that new BES choke transformer can meet the anti-interference requirements. Finally, the four-terminal network model of BES choke transformer is established, and the relevant fourterminal network parameters are obtained, which provides important theoretical basis for the establishment of the complete track circuit system model.

\section{Acknowledgement}

This work is supported by science and technology project of Shandong Provincial Transport Department under Grant No. 2019B66, and Shandong Jiaotong University fund under Grant No. Z201921.

\section{References}

[1] Xiaotao Tang. Problems and countermeasures of ZPW-2000 track circuit parameter adjustment in high-speed railway. Science and Technology Consultation Herald, 11, 70( 2014).
[2] China Railway Corporation. Technical principles for configuration and application of CTCS-2 train control system in high-speed railway.

[3] Shuqiang Zhao. Adjustment of polarity crossing for newly opened station track circuit. Selected papers of 2007 academic activity month of Henan Railway Association: China Railway Association, 196(2007).

[4] Shiwu Yang, Yilin Li, Haikang Chen. A scheme to reduce the potential difference at both ends of the insulating joint based on the simulation model of traction power supply system of typical high-speed railway stations. China Railway Science, 37(2016).

[5] Hongjun Bi, Xing Zhao, Minhui Zhang. Analysis of potential difference of mechanical insulating joint in high-speed railway station. Journal of Northern Jiaotong University, 37 (2013).

[6] Linhai Zhao, Lei Ren. Study on the influence of JTC adjacent section interference on TCR. Journal of railways, 35 (2013).

[7] Wentao Li . Study on the protection measures for the over area interference of the insulation damage signal of the integrated track circuit in the station. Railway communication signal, 54 (2018).

[8] Guoqing Li. Research on the influence of high-speed railway traction current on the track circuit in ZPW-2000 station. Jiaotong University, 2016.

[9] Shangpeng Sun, huibing Zhao, Dewang Chen. Research on calculation method of shunting resistance 
of track circuit based on electric contact theory. Journal of railways, $\mathbf{3 6}$ (2014).

[10] Jialiang Liu, Shiwu Yang, Canghai Ma. Study on four terminal network model of 1200A / 1400A choke adapter transformer under heavy load condition. Railway communication signal engineering technology, 11 (2014). 